\title{
Über einige neue Verbindungen von Stickstoff und Wasserstoff mit Metallen
}

\author{
(Vorläufige Mitteilung)
}

von

\section{F. W. Dafert und R. Miklauz.}

(Vorgelegt in der Sitzung am 24. Juni 1909.)

Nach den Beobachtungen von $\mathrm{H}$. Moissan ${ }^{1}$ beginnt Calciumnitrid, das im Wasserstoffstrom erhitzt wird, oberhalb Rotglut in Hydrid überzugehen. »Seine Farbe wird immer heller und wenn man die Temperatur steigert, nimmt die gebildete Menge von Hydrid $z u$. "Gelegentlich von Versuchen technischer Natur erschien es uns angezeigt, diesen Prozeß näher zu studieren und vor allem quantitativ zu verfolgen, ähnlich wie dies, freilich in anderer Richtung und zu anderen Zwecken, F. Haber und G. van Oordt' ${ }^{2}$ getan haben. Die letztgenannten Autoren fassen ihre Beobachtungen in den Satz zusammen: "Es wurde gezeigt, daß die umkehrbare Reaktion $\mathrm{Ca}_{3} \mathrm{~N}_{2}+3 \mathrm{H}_{2} \leftrightarrows 3 \mathrm{CaH}_{2}+\mathrm{N}_{2}$ sich bei Rotglut leicht verwirklicht und mit der Reaktion $\mathrm{N}_{2}+3 \mathrm{H}_{2} \leftrightarrows 2 \mathrm{NH}_{3}$ verknüpft. In Übereinstimmung mit der Theorie tritt bei der Einwirkung von Wasserstoff auf Calciumnitrid Ammoniak in nachweisbaren Mengen auf, bei der Einwirkung von Stickstoff auf Calciumhydrid aber nicht. Das Temperaturgebiet, in welchem die Calciumverbindungen als Zwischenkörper bei der Ammoniaksynthese aus den Elementen benützt werden können, liegt zu hoch, als daß erhebliche Ausbeuten an Ammoniak mit ihrer

1 H. Mo is san, Compt. rend., 127, 31 und 498 (1898).

2 F. Haber und G. van Oordt, Zeitschrift für anorgan. Chemie, 44 , 357 (1905). 
Hilfe erreichbar wären.« Bei der Behandlung von Nitrid mit Wasserstoff erhielten sie nicht mehr als 15 Teile Hydrid auf 100 Teile unveränderten Nitrids, und wenn sie Stickstoff auf das Hydrid einwirken ließen, ein Produkt, das eine ziemlich ähnliche Zusammensetzung hatte. Es gelang ihnen somit nicht, das Nitrid vollständig in Hydrid und umgekehrt, das Hydrid in Nitrid zu verwandeln.

Wir sind bei unseren Arbeiten, die - wie gleich hier betont sei - ungewöhnliche experimentelle Schwierigkeiten bieten und daher nur langsam fortschreiten, gleichfalls von der Annahme ausgegangen, daß es sich bei der Umwandlung des Calciumnitrids in Calciumhydrid tatsächlich um einen einfachen Austausch des Stickstoffs gegen Wasserstoff handelt, erkannten aber alsbald, daß dies nur bedingt richtig ist. Erhitzt man reines Calciumnitrid in vollkommen trockenem und von allen schädlichen Nebenbestandteilen befreitem Wasserstoffgas innerhalb bestimmter, ziemlich weiter Temperaturgrenzen (um $700^{\circ} \mathrm{C}$.), so tritt nicht die erwartete Gewichtsabnahme ein, sondern unter lebhafter Wasserstoffabsorption eine Gewichts zunah me, die bei unseren Versuchen von $2 \cdot 16$ bis $2 \cdot 84 \%$ schwankte. Daraus geht hervor, daß zunächst nicht das Calciumnitrid zersetzt wird, sondern, daß überraschenderweise eine Addition von Wasserstoff zum Calciumnitrid stattfindet. Hierbei entsteht, wie sich gezeigt hat, eine ganz neue chemische Verbindung, deren äußere Merkmale sehr charakteristisch sind. Ihre Farbe ist im Unterschiede vom Hydrid und dem dunkelrotbraunen Nitrid zitronen-bis or ang egelb, ein Umstand, der die von früheren Beobachtern ${ }^{1}$ bemerkte Gelbfärbung des Nitrids im Wasserstoffstrom bei Rotglut erklärt. Die Substanz verändert sich in der Nähe der Bildungstemperatur, soweit unsere Hiffsmittel dies bis jetzt festzustellen erlaubten, weder durch Erhitzen im Wasserstoffstrom, noch durch die Einwirkung von Stickstoff in nennenswertem Ausmaß, zeichnet sich aber durch eine merkwürdige Eigenschaft, durch starke Lichtempfindlichkeit, aus. Das zerstreute Tageslicht verwandelt sie nach und nach, das Sonnenlicht sehr rasch ohne merkliche Gewichtsveränderung in einen

1 F. Haber und G. van Oordt, a. a. O., p. 363. 
grauschwarzen Körper, aus dem beim Erwärmen im Stickstoffstrom anscheinend die ursprüngliche gelbe Substanz regeneriert wird. Mit Wasser bildet sich Ammoniak und Wasserstoffgas. Im Sauerstoffstrom verbrennt diese Verbindung nur schwierig und unvollkommen, so daß die Elementaranalyse auf bisher noch nicht überwundene Hindernisse stößt. Wir mußten uns damit begnügen, den Calciumgehalt direkt zu ermitteln und zur vorläufigen Beurteilung der Zusammensetzung die Werte heranzuziehen, welche sich für den Stickstoff aus der Menge des bei der Behandlung der Substanz mit Wasser abgespalteten Ammoniaks und für den Wasserstoff aus der Gewichtszunahme berechnen. Mit den gasvolumetrisch gefundenen Zahlen für Wasserstoff läßt sich nicht gut operieren, weil sie je nach der unbekannten Konstitution der betreffenden Substanz entweder zur Hälfte oder voll in Rechnung zu setzen sind. ${ }^{1}$ Auf diesem Wege gelangen wir zu dem Schlusse, daß die gelbe Substanz durchschnittlich enthält:

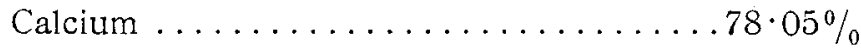

Stickstoff . . . . . . . . . . . . . . 16 66

Wasserstoff . . . . . . . . . . . 2 50

Eisen, Silicium, Kohle, Sauerstoff usw. . . . . $2 \cdot 79$

Daraus berechnet sich für die reine Substanz:

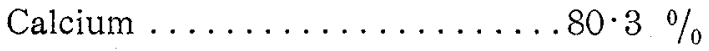

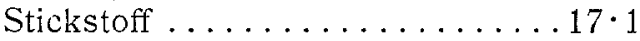

Wasserstoff ........... $2 \cdot 6$.

Ganz ähnliche Werte ergeben sich für das schwarze Umwandlungsprodukt.

Es wäre verfrüht, aus diesen provisorischen Zahlen endgültige Schlüsse auf die Konstitution ziehen zu wollen; immerhin gestatten sie, uns eine Vorstellung von den Vorgängen zu bilden, welche sich möglicherweise hier abspielen. Die Zusammen-

1 Wie sich F. Haber und van Oordt mit diesem Umstande abgefunden haben, ist nicht recht ersichtlich. Sie berechnen ihre Wasserstoffzahlen nach der Gleichung $\mathrm{CaH}_{2}+\mathrm{H}_{2} \mathrm{O}=\mathrm{CaO}+\mathrm{H}_{4}$, während doch auch $\mathrm{Ca}_{3}\left(\mathrm{NH}_{2}\right)_{2}+3 \mathrm{H}_{2} \mathrm{O}$ $=3 \mathrm{CaO}+2 \mathrm{NH}_{3}+\mathrm{H}_{4}$, ja sogar $\mathrm{CaNH}+\mathrm{H}_{2} \mathrm{O}=\mathrm{CaO}+\mathrm{NH}_{3}$ in Betracht kommt. 
setzung entspricht am besten der Formel $\mathrm{Ca}_{3} \mathrm{~N}_{2} \mathrm{H}_{4}$ mit folgenden theoretischen Gehalten:

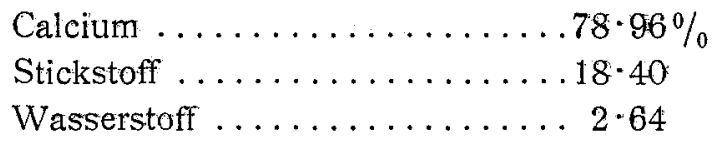

Es würde sich demnach, wofür die glatte Addition spricht, zunächst aus dem Calciumnitrid ein auch theoretisch interessantes, in der Hitze beständiges Tricalciumamid bilden:

$$
\mathrm{Ca}_{3} \mathrm{~N}_{2}+\mathrm{H}_{4}=\mathrm{Ca}_{3}\left(\mathrm{NH}_{2}\right)_{2} \text {. }
$$

Letzteres zerfällt im Sonnenlicht in Calciumimid und Calciumhydrid:

$$
\mathrm{Ca}_{3}\left(\mathrm{NH}_{2}\right)_{2}=2 \mathrm{CaNH}+\mathrm{CaH}_{2} \text {. }
$$

Zu Gunsten der Annahme, daß ein solches Tricalciumamid existiert und eine - unter den Bedingungen unserer Versuche -- sehr feste Verbindung darstellt, spricht das Verhalten des Hydrids gegen Stickstoff. Leitet man Stickstoff über Calciumhydrid, so nimmt dessen Gewicht unter Wasserstoffabspaltung beträchtlich zu und es bildet sich eine in ihren äußeren Merkmalen nach mit dem aus Nitrid hergestellten Produkt sehr ähnliche oder identische Substanz, ebenso wenn man Gemenge von Wasserstoff und Stickstoff auf metallisches Calcium einwirken läßt. Die genaue Ermittlung der Gewichtszunahme ist uns aber in diesen Fällen noch nicht gelungen, sicher konnten wir nur beobachten, daß die Zunahme beim Calciumhydrid nicht unter $17 \%$ beträgt, während die Theorie $20.6 \%$ erfordert.

Um in einem gewissen Sinne die Richtigkeit der gemachten Beobachtungen $\mathrm{zu}$ überprüfen, studierten wir das Verhalten anderer Metallnitride gegen Wasserstoff. Magnesiumnitrid zeigt keine Neigung, Wasserstoff zu addieren, dafür fanden wir eine um so größere bei Lithiumnitrid. Nach L. Ouvrard ${ }^{1}$ entwickelt Lithiumnitrid beim Erhitzen im Wasserstoffstrom Ammoniak, Lithiumhydrid liefert dagegen nach Guntz ${ }^{2}$ beim Behandeln

1 L. Ouvrard, Compt. rend., 114,121 (1892).

2 Guntz, ebenda, 122, 146 (1896), und 123, 997 (1896). 
mit Stickstoff unter Wasserstoffentwicklung Nitrid. Guntz schließt aus seinen Beobachtungen über die Bildungswärmen des Lithiumnitrids und -hydrids: »que l'hydrogène doit décomposer l'azoture de lithium et c'est ce que l'on constate assez facilement en chauffant $\mathrm{Li}_{3} \mathrm{Az}$ dans un courant d'hydrogène, car on obtient de l'hydrure et de lithium faisant effervescence avec l'eau en dégageant de l'hydrogène. On peut cependant obtenir la réaction inverse et décomposer $\mathrm{LiH}$ dans un courant d'azote, mais cette réaction tient à la dissociation à haute temperature de l'hydrure de lithium chauffé dans un courant d'azote qui se combine en métal devenu libre." Alle diese Angaben bedürfen der Ergänzung und Berichtigung.

Das schwarzbraune Lithiumnitrid nimmt beim Erwärmen im Wasserstoffstrom unter bestimmten Verhältnissen sehr lebhaft Wasserstoff auf, und zwar durchschnittlich $5.40 \%$, umgekehrt addiert Lithiumhydrid mindestens $51 \cdot 09 \%$ Stickstoff. Die entsprechenden weißen Additionsprodukte zeigen in ihrem Verhalten unleugbare Ähnlichkeit mit jenen des Calciums. Der Farbenumschlag erfolgt hier von weiß über rosa nach rot, und zwar noch schneller als beim Calcium. Die Regenerierung der weißen Form aus der roten ist uns bisher nicht gelungen, wohl aber haben wir festgestellt, daß ein erheblicher Anteil des in der letzteren enthaltenen Wasserstoffs bei der Behandlung mit Wasser nich $\mathrm{t}$ als solcher in Freiheit gesetzt wird, also vermutlich Imidwasserstoff ist. Die aus dem Lithiumnitrid entstehende Verbindung muß etwa $5 \cdot 2 \%$ Wasserstoff enthalten, was wiederum am besten mit dem Trilithiumamid $\mathrm{Li}_{3} \mathrm{NH}_{2}$ (Theorje $5 \cdot 4 \%$; Zunahme bei der Bildung aus dem Hydrid $54 \cdot 1 \%$ ) übereinstimmt. Die Elementaranalyse bietet in diesem Falle weniger Schwierigkeiten, um so größere die Darstellung einigermaßen reiner Präparate, weil Lithium und Lithiumhydrid alle Gefäße heftig angreifen und man nur unter Anwendung gewisser Kunstgriffe dem Ziele näher kommt. Das Verhalten des hypothetischen Trilithiumamids gegen Licht läßt sich am einfachsten ebenfalls als Imidbildung auffassen: $\mathrm{Li}_{3} \mathrm{NH}_{2}=\mathrm{Li}_{2} \mathrm{NH}+\mathrm{LiH}$. Die intensiv rote Verbindung wäre das bisher unbekannte Lithiumimid $\mathrm{Li}_{2} \mathrm{NH}$ in Mischung mit Lithiumhydrid, Fragen, auf die wir selbstverständlich seinerzeit ausführlich zurückkommen werden. 
654 F.W.Dafert u. R. Miklauz, Wasserstoff- und Stickstoffverb. ete.

Wir haben Grund zur Annahme, daß auch andere Metallnitride sich analog verhalten und daß sich das von uns einstweilen als Trilithiumamid angesprochene Additionsprodukt des Wasserstoffs zum Lithiumnitrid, seiner großen Reaktionsfähigkeit halber, als Mittel zur Herstellung direkt nicht erhältlicher Hydronitride eignet. Zweck dieser vorläufigen Mitteilung ist, uns das einschlägige Arbeitsgebiet zu sichern. 